\title{
Assessment of Type of Mucosal Response in Gallstone Patients Undergoing Laparoscopic Cholecystectomy: A Prospective Study
}

\author{
Ashwani Kumar ${ }^{1}$, Pandey Satish Chandra ${ }^{1}$ \\ Associate Professor, Department of Surgery, Mayo Institute of Medical Sciences, Barabanki, Uttar Pradesh, India. \\ Corresponding author: Pandey Satish Chandra, Associate Professor, Department of Surgery, Mayo Institute of Medical \\ Sciences, Barabanki, Uttar Pradesh, India
}

DOI: $10.21276 /$ ijcmsr.2018.3.2.3

How to cite this article: Ashwani Kumar, Pandey Satish Chandra. Assessment of type of mucosal response in gallstone patients undergoing laparoscopic cholecystectomy: a prospective study. International Journal of Contemporary Medicine Surgery and Radiology. 2018;3(2):B8-B10.

\section{A B S T R A C T}

Introduction: Gallstones are becoming increasingly common; they are seen in all age groups, but the incidence increases with age. Gallstone disease produces diverse histopathological changes in gallbladder mucosa-namely, acute inflammation, chronic Inflammation, granulomatous inflammation, hyperplasia, cholesterolosis, dysplasia and carcinoma. Hence; we planned the present study to assess various responses of the gallbladder mucosa with the different characteristic of gall stones.

Material and methods: The present study included evaluation of various changes occurring in gallbladder mucosa in patients undergoing cholecystectomy. The present study included histopathological examination of 50 gall stone patients who underwent cholecystectomy. All the sections were stained with $\mathrm{H} \& \mathrm{E}$ staining and were analyzed by two independent pathologists. Morphological characteristic of the gallstones were characterized on the basis of criteria given previously in the literature. All the observations were recorded on excel sheet and were evaluated with SPSS software.

Results: A total of 50 patients undergoing laparoscopic cholecystectomy were included in the present study. Out of 50 patients, in 80 percent of the patients, the stones were of mixed or combined type. Mean size of stones in patients with histopathologic diagnosis was $3.98 \mathrm{~cm}$ while in patients with hyperplasia and cholecystitis with metaplasia, the size of stone was found to be 1.5 and $0.85 \mathrm{~cm}$ respectively.

Conclusion: Size of stone significantly correlates with the type of mucosal response in gallstone patients undergoing laparoscopic cholecystectomy.

Key words: Gallstones, Hyperplasia, Mucosal

\section{INTRODUCTION}

Gallbladder is an accessory organ of the digestive tract, storing and concentrating bile between meals. Gallstones are becoming increasingly common; they are seen in all age groups, but the incidence increases with age; and about a quarter of women over 60 years will develop them. Cholelithiasis has been described as a disease of civilization. ${ }^{1-3}$ Gallstone disease produces diverse histopathological changes in gallbladder mucosa-namely, acute inflammation, chronic Inflammation, granulomatous inflammation, hyperplasia, cholesterolosis, dysplasia and carcinoma. ${ }^{4-6}$ Authors from one of the past study, evaluated 1101 patients with symptomatic cholecystitis who underwent elective cholecystectomy along with intraoperative liver biopsy. In 96.7 percent of the patients, on histologic examination, inflammatory changes were seen. In 27.9 percent of the patients, clinical alterations in the liver parenchymal were present. Under the lights of results, they concluded that cholecystectomy is the treatment of choice as majority of cases of cholelithiasis is of inflammatory type. Under the light of above mentioned data, we planned the present study to assess various responses of the gallbladder mucosa with the different characteristic of gall stones.

\section{MATERIAL AND METHODS}

The present study was planned in the department of general surgery and pathology of the medical institute and it included evaluation of various changes occurring in gallbladder mucosa in patients undergoing cholecystectomy.

Ethical approval was obtained from institutional ethical committee and written consent was obtained from all the patients after explaining in detail the entire research protocol. This was a prospective study and a total of 100 patients were enrolled in it.

Inclusion criteria for the present study included:

- Patients undergoing cholecystectomy for symptomatic cholecystitis due to cholelithiasis

- Patients with negative history of any other systemic illness

- $\quad$ Patients with negative history of any known drug allergy The present study included histopathological examination 
of 100 gall stone patients who underwent cholecystectomy. The tissue was properly sampled and processed by routine histological techniques for paraffin embedding and sectioning at 4 micron thickness. Four sections including entire wall were obtained: two from body, one each from fundus and neck of the gall bladder. All the sections were stained with $\mathrm{H} \& \mathrm{E}$ staining and were analyzed by two independent pathologists. Morphological characteristic of the gallstones were characterized on the basis of criteria given previously in the literature. ${ }^{8}$

\section{STATISTICAL ANALYSIS}

All the observations were recorded on excel sheet and were evaluated with SPSS software. Chi- square test and student $t$ test were used for assessment of level of significance. P-value of less than 0.05 was taken as significant.

\section{RESULTS}

\begin{tabular}{|l|c|c|}
\hline Age group & Frequency & Percent \\
\hline$<25$ & 12 & 12 \\
\hline $26-35$ & 20 & 20 \\
\hline $36-45$ & 30 & 30 \\
\hline $46-55$ & 20 & 20 \\
\hline$>55$ & 18 & 18 \\
\hline Total & 100 & 100 \\
\hline \multicolumn{2}{|c|}{ Table-1: Distribution of subjects according to age group } \\
\hline
\end{tabular}

\begin{tabular}{|l|c|c|}
\hline Type & Frequency & Percent \\
\hline Mixed & 50 & 50 \\
\hline Combined & 30 & 30 \\
\hline Cholesterol & 10 & 10 \\
\hline Pigment & 10 & 10 \\
\hline Total & 100 & 100 \\
\hline \multicolumn{2}{|c|}{ Table-2: Distribution of subjects according to type of stone } \\
\hline
\end{tabular}

\begin{tabular}{|c|c|c|c|}
\hline Diagnosis & $\begin{array}{l}\text { Mean } \\
\text { size of } \\
\text { stone } \\
(\mathrm{cm})\end{array}$ & t- value & $p$-value \\
\hline Cholecystitis & 0.72 & \multirow[t]{4}{*}{10.896} & \multirow[t]{4}{*}{0.012} \\
\hline Hyperplasia & 1.50 & & \\
\hline Cholecystitis with metaplasia & 0.85 & & \\
\hline Carcinoma & 3.98 & & \\
\hline
\end{tabular}

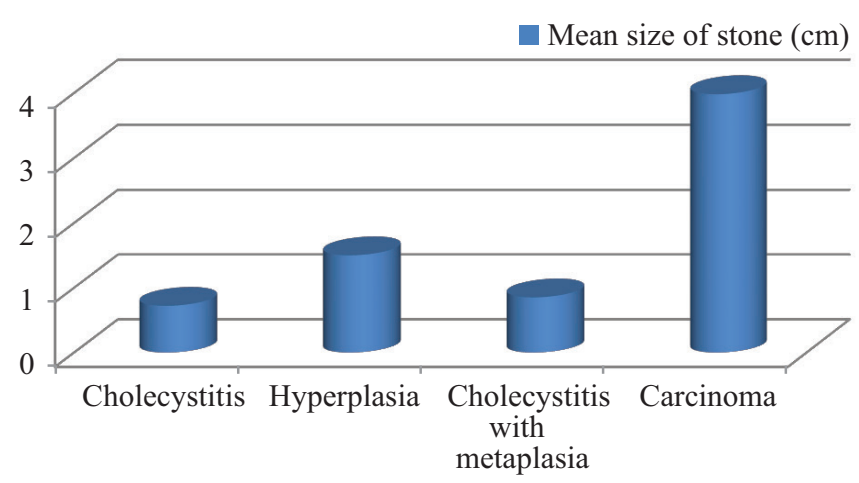

Graph-1: Type of mucosal response with mean size of stone
A total of 100 patients undergoing laparoscopic cholecystectomy were included in the present study. Among these 100 patients, majority were in between age group of 40 years and above. Out of theses 100 patients, 80 patients were females and the remaining 20 were males. Out of 100 patients, in 80 percent of the patients, the stones were of mixed or combined type. In 5 patients each, the stones were of cholesterol and pigmented type respectively. Mean size of stones in patients with histopathologic diagnosis was $3.98 \mathrm{~cm}$ while in patients with hyperplasia and cholecystitis with metaplasia, the size of stone was found to be 1.5 and $0.85 \mathrm{~cm}$ respectively. Significant results were obtained while correlating the size of the stone with the type of mucosal response.

\section{DISCUSSION}

In the present study, we observed significant results while correlating the size of stone with type of mucosal response. In another previous report, Terada $\mathrm{T}$ et al described the morphologies and incidence of gall bladder diseases and pathologies of 540 cholecystectomies. Mean age of the patients of their study was 64.75 years. In their study, they observed a female preponderance. More than 95 percent of the patients of their study had gallstones. Acute cholecystitis was found to be present in one and a half percent of the patients, while chronic cholecystitis was found to be present in 94.1 percent of the patients. Approximately two percent of the patients of their study were suffering from adenocarcinomas, while on case of cystadenocarcinoma was present. They observed that only 2 percent of the patients had normal gallbladder mucosa. Florid occurrence of epithelial proliferation in adenomyomatous changes were found only in few cases. However; among those cases, they didn't observed any perineural invasion. They also didn't recognize any form of perineural invasion in pyloric gland metaplasia cases. Among the seven cases of adenoma present in their study, all were of intestinal type. In their series, there were no cases of heterotipc tissue, intestinal metaplasia, intraepithelial neoplasm, and other malignancies. ${ }^{9}$

Yaylak F et al (2014) evaluated the association between cholesterolosis and the reports indicating some histopathological alterations in symptomatic cholecystitis. Histopathological reports of 432 cholecystectomy specimens between January 2011 and June 2013 were reviewed. Three reports were excluded due to perioperative diagnosis of cancer. Reports of 429 cholecystectomy specimens of the acute and symptomatic chronic cholecystitis patients were analyzed. Standardization of the reporting was questioned. Age, gender, histopathological wall thickness of gallbladder, reporting rates of acute inflammation, cholesterolosis, polypoid lesions, epithelial hyperplasia, gastric or intestinal metaplasia, dysplasia and incidental cancer were investigated and compared between patients with and without cholesterolosis. Reported rates of histopathological findings were comparable between patients under and over 60 years old and patients with and without reported cholesterolosis. Reported histopathological findings were presented as acute inflammation in 46 (10.7\%), cholesterolosis in 79 (18.4\%), gallbladder polypoid lesions in 7 (1.6\%), epithelial hyperplasia 
in $16(3.7 \%)$, metaplasia of any type in $34(7.9 \%)$ of 429 patients. Dysplasia was excluded whereas one incidental gallbladder carcinoma was reported. Epithelial hyperplasia and metaplasia were found to be related to age. Gallbladder wall thickness was decreased with cholesterolosis. However, only a correlation between cholesterolosis and gender or metaplasia was noted. Recent study suggested that cholesterolosis is somehow associated with metaplasia. ${ }^{10}$ Goyal S et al (2014) correlate the various types of mucosal response like inflammation, hyperplasia, metaplasia, and carcinoma to different gall stones characteristics (like number and morphology type). A retrospective study of 346 cases of removed GB was done which was based on the histological changes. A total of $313(90 \%)$ were associated with gallstones and the rest 33 cases (10\%) were of acalculous cholecystitis. The changes in mucosa of calculous GB (313 cases) were studied and correlation between the mucosal changes and the number and type of stones was evaluated. Varied parameters of gall stones like number and morphological type were studied. Tissue section for histopathological studies was taken from fundus, body, neck, and from abnormal looking areas while doing grossing. Gender study revealed a higher incidence of inflammatory changes in males, while GB hyperplasia, intestinal metaplasia, and cancer were only found in females. A progressive increase in the average age was noticed from intestinal metaplasia to carcinoma, suggesting that the metaplasia-carcinoma succession may occur in GB cancer. Correlation of mucosal changes with size and morphological type of stones has suggested that there could be an association between some histological alterations of GB and cancer. ${ }^{11}$

\section{CONCLUSION}

From the above results, the authors concluded that size of stone significantly correlates with the type of mucosal response in gallstone patients undergoing laparoscopic cholecystectomy.

\section{REFERENCES}

1. Housset C, Chrétien Y, Debray D, Chignard N. Functions of the Gallbladder. Compr Physiol. 2016;6(3):1549-77.

2. Stinton LM, Shaffer EA. Epidemiology of Gallbladder Disease: Cholelithiasis and Cancer. Gut and Liver. 2012;6(2):172-187.

3. Mathur SK, Duhan A, Singh S, Aggarwal M, Aggarwal G, Sen R, Singh S, Garg S.Correlation of gallstone characteristics with mucosal changes in gall bladder. Trop Gastroenterol. 2012;33(1):39-44.

4. Njeze GE. Gallstones. Nigerian Journal of Surgery: Official Publication of the Nigerian Surgical Research Society. 2013;19(2):49-55.

5. Baidya R, Sigdel B, Baidya NL. Histopathological changes in gallbladder mucosa associated with cholelithiasis. Journal of Pathology of Nepal 2012;2(1);224 - 225.

6. Baig SJ, Biswas S, Das S, Basu K, Chattopadhyay G. Histopathological changes in gallbladder mucosa in cholelithiasis: correlation with chemical composition of gallstones. In a previous study, authors Trop
Gastroenterol. 2002;23(1):25-7.

7. Badke A, Schwenk W, Böhm B, Stock W. Histopathological changes of gallbladder and liver parenchyma in symptomatic cholelithiasis. Dtsch Med Wochenschr. 1993;118(22):809-13.

8. Mohan H, Punia RP, Dhawan SB, Sekhon MS. Morphological spectrum of gallstone disease in 1100 cholecystectomies in north India. Indian J Surg 2005;67:140-2.

9. Terada T1. Histopathologic features and frequency of gall bladder lesions in consecutive 540 cholecystectomies. Int J Clin Exp Pathol. 2013;6(1):91-6.

10. Yaylak F1, Deger A2, Ucar BI3, Sonmez Y4, Bayhan Z5, Yetisir F6. Cholesterolosis in routine histopathological examination after cholecystectomy: what should a surgeon behold in the reports? Int J Surg. 2014;12(11):1187-91.

11. Goyal S, Singla S, Duhan A. Correlation between gallstones characteristics and gallbladder mucosal changes: A retrospective study of 313 patients. Clin Cancer Investig J. 2014;3(2):157-61.

Source of Support: Nil; Conflict of Interest: None

Submitted: 20-03-2018; Published online: 25-04-2018 